\title{
Critical Media. Proposals to articulate and activate devices of territorial transformation.
}

\section{SIGRADI2018 TECHNOPOLITICAS \\ xxii congresso da sociedade iberoamericana de gráfica digital 22th conference of the iberoamerican society of digital graphics $07|08| 09 \mid$ novembro|2018 iau usp | são carlos | sp br}

\author{
María Elena Tosello \\ Universidad Nacional del Litoral | Argentina | maritosello@gmail.com \\ María Georgina Bredanini Colombo \\ Universidad Nacional del Litoral | Argentina | gbredani@fadu.unl.edu.ar \\ Cecilia Verónica Zorzón \\ Universidad Nacional del Litoral | Argentina | ceciliazorzon@gmail.com \\ Marcelo Fabián Jereb \\ Universidad Nacional del Litoral | Argentina | marcelojereb@gmail.com
}

\begin{abstract}
This paper discusses the aims of media and technologies from a political insight, knowing the shortcomings and contradictions that cross the Latin American countries, for the purpose of being able to visualize them, think them and provide possible solutions, taking advantage of the creative potential of the university. With this objective, we developed an experience that integrated a research project on the design and production of artifacts, interfaces and representations capable of articulating the links between subjects, actions and dimensions, with the teaching and learning processes of an interdisciplinary design workshop, that created and activated territorial transformation devices with a sense of community collaboration.
\end{abstract}

Keywords: Digital Media; Critical Theory; Participative Design; Collaborative Design; Learning Process.

\section{INTRODUCCIÓN}

Cuando las computadoras personales comenzaron a popularizarse, hace aproximadamente 30 años atrás, la educación, investigación y producción con medios digitales en general, se fundamentaba y enfocaba en objetivos técnicos. En las facultades de arquitectura, especialistas en informática dictaban cursos de CAD dirigidos a la adquisición de habilidades para el manejo de los softwares más utilizados. Con el tiempo, además de los objetivos instrumentales, comenzaron a incorporarse aplicaciones de estas "nuevas tecnologías" (que aún no eran "nuevos medios") en distintas tareas de la actividad proyectual, en la mayoría de los casos como sistema de representación más eficaz, para "pasar en limpio" el proyecto; en otros, como instrumento para lograr una mejor percepción y compresión del espacio (Mitchell, 1990; Montagú, 1996; Tosello, 1997); y en algunas ocasiones, como protagonista y participante activo del proceso de diseño, afectando y transformando profundamente tanto los procedimientos como los resultados del mismo (Novak, 1991; Bermúdez, 1995; Combes, 1997). En la actualidad, en algunos ámbitos educativos aún persiste está tendencia que se manifiesta a través de métodos de modelado paramétrico, BIM y fabricación digital.

Una asignatura pendiente por muchos años fue la relación entre los medios digitales y la filosofía, encuentro que en nuestro contexto recién se concretó en el año 2000, a través de las teorías del posestructuralismo francés representado por Derrida, Deleuze y Foucault (Levy, 1998; Senegala, 1999; Tosello, 2002). El fructífero diálogo con la filosofía otorgó contenido y espesor simbólico a las operaciones digitales, superando el estadio meramente instrumental, y ubicó a la mediatización de los procesos proyectuales en un contexto más amplio, el de una época de cambio de paradigmas epistemológicos y culturales.

\section{INTERESES TÉCNICOS Y PRÁCTICOS}

Estas fases son descriptas claramente por la Teoría Crítica como distintos modos de razonamiento que influyen sobre el interés cognoscitivo en la construcción de significados y en la vida social: el instrumental o interés técnico, y el interpretativo o interés práctico (Habermas, 1989). Según Marcuse (1993), el interés técnico-instrumental supone un hombre unidimensional y consumista, anestesiado por los mass media que impiden la reflexión y el pensamiento crítico. El modelo de racionalidad técnica -que se basa en teorías positivistas y se propone como instrumento de dominación- plantea una relación utilitaria con el mundo, transformando la naturaleza sin valores ni principios éticos.

El interés práctico comprende los modelos de interacción comunicativa y simbólica que dan forma al individuo, el cual se considera un factor activo de producción e interpretación de significados que lo hacen consciente del mundo en el que vive, para tomar posición respecto a los fines y las reglas del pensamiento, porque así como las 

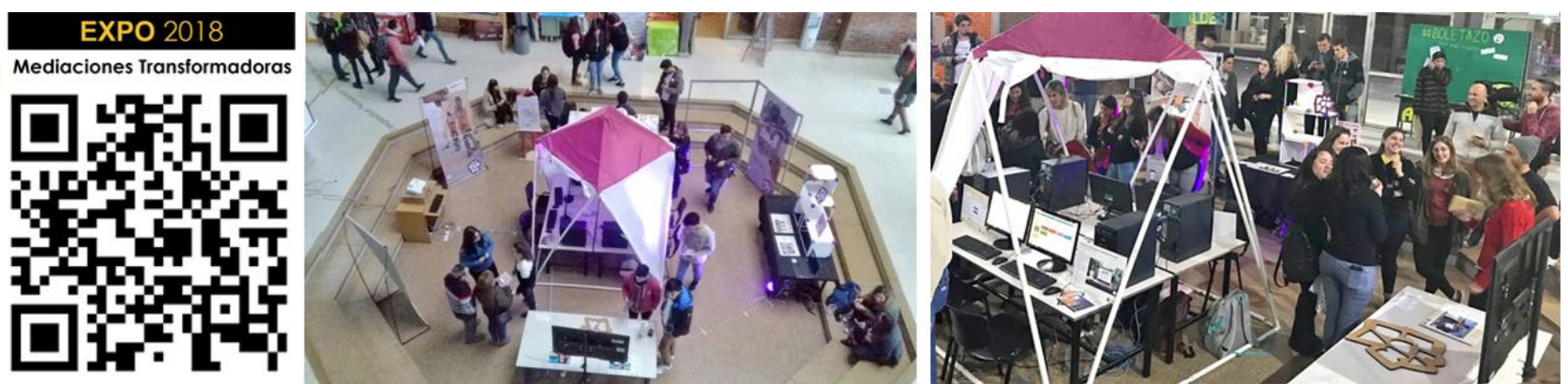

Figura 1: Exposición del Taller de Gráfica Digital "Mediaciones Transformadoras". Muestra colectiva en el hall central de la Facultad de Arquitectura, Diseño y Urbanismo de la Universidad Nacional del Litoral. Fuente: autores.

tecnologías no son neutrales (Kranzberg, Davenport, [1972] 1979), los conocimientos tampoco lo son, sino que éstos pueden tener diferentes finalidades. De esta manera, el conocimiento se convierte en un acto social. El interés o razón práctica se expresa como saber o como ciencia y, aunque adquiere una dimensión social, es insuficiente para consolidar la libertad como conducta humana con características de autonomía e independencia, ya que no aporta las condiciones necesarias para alcanzarlas.

\section{RAZÓN CRÍTICA}

A los intereses técnicos y prácticos, Habermas incorpora el interés emancipatorio o razón crítica, partiendo de la crítica a las Ciencias Naturales, en las cuales el objeto de estudio está separado del sujeto, y considerando que en las Ciencias Sociales y Humanas, el objeto es el sujeto.

Reinterpretando la distopía de Huxley (1932) [2014], analiza la fractura de la ciencia positivista frente "al mundo en que viven los hombres", y dado que concibe al hombre como parte indisoluble de la naturaleza, propone a la crítica creativa como instrumento para recuperar la reflexión y reencontrarnos con la naturaleza y el mundo.

El razonamiento crítico, característico de todo proceso reflexivo, permite cuestionar el mundo que habitamos e imaginar otras realidades posibles. Se trata de un sistema evolutivo con capacidad de dudar de la realidad y el orden dados, que favorece la conciencia de sí mismo (autoconocimiento), y la acción responsable y autónoma, las cuales -según Habermas- llevarían a la emancipación en la medida en que comprendamos las condiciones sociopolíticas y las relaciones de poder.

Como "las tecnologías no dispensan al hombre de la acción", la técnica debe complementarse con la política para la reflexión y determinación de los fines prácticos, en una democracia entendida como "forma institucionalmente asegurada de una comunicación general y pública que se ocupa de las cuestiones prácticas" (Habermas, Óp. Cit.). En tanto alternativa a la razón técnico-instrumental, en este modelo el conocimiento se configura mediante procesos de interacción social basados en Acciones Comunicativas y Mediaciones del Lenguaje.

\section{MEDIACIONES DEL ESPACIO AUMENTADO}

El proyecto de investigación "Mediaciones del Espacio Aumentado", propone diseñar y producir artefactos, interfaces y representaciones capaces de articular los vínculos entre sujetos, acciones y dimensiones, favoreciendo la comunicación y posibilitando usos emergentes y múltiples sentidos para habitar inclusivamente un espacio-tiempo heterogéneo.

Conociendo las insuficiencias y contradicciones que atraviesan a los países latinoamericanos en diferentes dimensiones (desigualdades ambientales, económicas, sociales, etc.), y con el fin de poder visibilizarlas, pensarlas y reducirlas aprovechando el potencial creativo de la universidad con un sentido de comunidad, integramos el proyecto de investigación con los procesos de enseñanza y aprendizaje del Taller de Gráfica Digital TGD-, alentando la transferencia de los conocimientos al medio social. Con este objetivo, se orientó su acción a la articulación con el territorio, enfocándose en aportar posibles soluciones a problemas concretos de la Asociación Civil La Funda, un espacio socioeducativo dedicado a proteger y acompañar las infancias vulnerables de barrios periféricos de la ciudad de Santa $\mathrm{Fe}$, que pone énfasis en la nutrición comunitaria, la educación sexual integral y la educación popular, desde la perspectiva de la salud pública y los derechos humanos.

\section{METODOLOGÍA}

En reuniones de cátedra previas al inicio del cuatrimestre, los responsables de la Asociación Civil informaron sobre las problemáticas de la institución y se previsualizaron posibles aportes que TGD podía realizar a través de prácticas o proyectos integrados a dispositivos de transformación territorial. Esta estrategia iba más allá de los tradicionales ejercicios desarrollados en los talleres proyectuales, ya que en esta propuesta los estudiantes se enfrentarían a situaciones reales.

Durante ese período, además, se definieron los recursos didácticos y las actividades a desarrollar (clases teóricas y prácticas, correcciones grupales, trabajos de campo, instancias de presentación y exposición de las producciones de los estudiantes), en relación a los contenidos, los objetivos perseguidos y el andamiaje teórico, así como también, las características del proyecto colaborativo a abordar durante todo el cuatrimestre y los criterios e instrumentos de evaluación del mismo.

Entre las pautas predefinidas, se decidió que el proyecto se organizaría en grupos interdisciplinarios de 3 a 5 estudiantes, para la elaboración de distintas propuestas que pudieran contribuir a resolver las problemáticas de la citada institución. En grupos interdisciplinarios, cada integrante realiza aportes diferentes, y los conocimientos que un estudiante comparte, enriquecen y traccionan a los demás. Se estimó que 1 de cada 5 estudiantes tendría acceso a hardware, softwares y servicios para realizar las actividades del taller. También se dispuso del FabLab de 


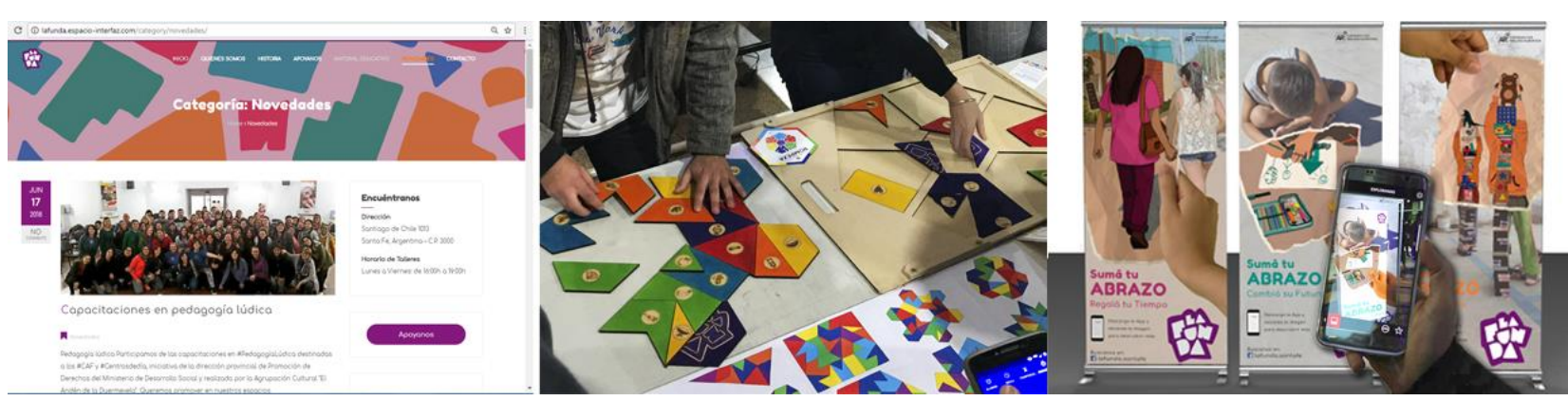

Figura 2: De izquierda a derecha: Web Institucional (G1); Rompecabezas didáctico (G2); Banners con Realidad Aumentada (G3).

la facultad con recursos tecnológicos y constructivos (PC, impresoras 3D y cortadoras laser).

Asimismo, se establecieron los medios y plataformas que integrarían la propuesta didáctica transmedia: grupo cerrado de Facebook para la comunicación entre estudiantes y docentes, mensajería instantánea a través de un grupo de WhatsApp para comunicación interna de la cátedra, plataformas de almacenamiento como medio para compartir los recursos (Google Drive), "Entorno Virtual de la Universidad Nacional del Litoral" (plataforma educativa Moodle) como espacio de debate y socialización de conocimientos, canal de YouTube y SlideShare como repositorios de material didáctico (tutoriales, clases, ejemplos, etc.), y enlaces a contenidos relacionados.

\section{DESARROLLO DE LA EXPERIENCIA}

Iniciamos la experiencia con clases teóricas que ofrecieron a los estudiantes un panorama general de la materia (abordaje teórico, contenidos, objetivos), y presentamos el trabajo práctico. A partir de charlas a cargo de representantes de la institución, donde comentaron sus proyectos, inquietudes y problemáticas, identificamos colaborativamente las principales necesidades: la falta de espacio y recursos, la organización de los voluntarios, el seguimiento de los niños/as, la difusión de actividades, la acumulación de residuos en el barrio, la violencia, etc.

Luego, mediante clases prácticas, investigamos los medios y tecnologías disponibles, y cruzando los problemas con los medios se formularon las propuestas, concebidas a través de la asociación y negociación entre sujetos e instituciones (Scolari, 2008).

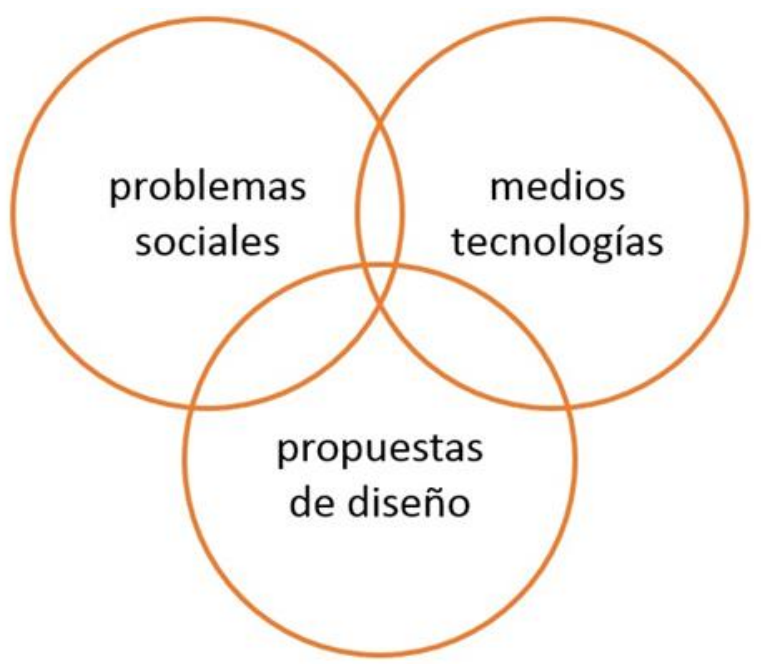

Cada grupo interdisciplinario de estudiantes trabajó sobre alguno de los problemas identificados y realizó una contribución diferente, aunque integrada a un proyecto general, explorando la capacidad de la Arquitectura y el Diseño para generar propuestas que reduzcan las asimetrías sociales, resignificando las finalidades de los instrumentos tecnológicos (Machado, 2000) y desviándolos de sus funciones predefinidas para producir una diferencia con un sentido político (Kozak, 2011).

Una vez que los estudiantes presentaron las ideas con las que intentarían dar solución a los problemas manifestados, comenzamos a dictar clases teóricoprácticas orientadas a las temáticas, recursos y técnicas particulares a abordar por los diferentes grupos. Éstos se distribuyeron en tres áreas: diseño y programación web; diseño paramétrico y fabricación digital; y producción de video y sonido, no obstante, algunos grupos plantearon temáticas transversales que involucraban múltiples áreas.

Durante todo el cuatrimestre se intercalaron las clases generales, con las correcciones y clases específicas para grupos reducidos. Asimismo, se fueron diseminando progresivamente los contenidos por las distintas plataformas, motivando la migración mediática a través de la concreción de instancias obligatorias (entrega de avances y participación en foros). Paralelamente, se realizaban interacciones en Facebook, donde se respondían inquietudes de los estudiantes, se establecían intercambios multidireccionales de información y se cargaban materiales de manera colaborativa.

A mitad del cuatrimestre, estudiantes y docentes efectuamos una visita extracurricular a los espacios físicos donde la Asociación Civil desarrolla sus actividades. En esta instancia, los grupos pudieron tomar contacto directo con la problemática y realizar un relevamiento fotográfico y audiovisual de las instalaciones, el contexto y sus habitantes, participando además de una experiencia lúdica conjunta. Todo el material obtenido fue cargado por estudiantes y docentes en una carpeta compartida de Google Drive que sería utilizada para el desarrollo de las propuestas.

En la entrega final, los grupos realizaron exposiciones orales donde explicaron el problema detectado, los objetivos que tuvieron en cuenta al plantear sus propuestas proyectuales y los diferentes procesos desarrollados, presentando los resultados alcanzados a través de producciones físicas o virtuales. 

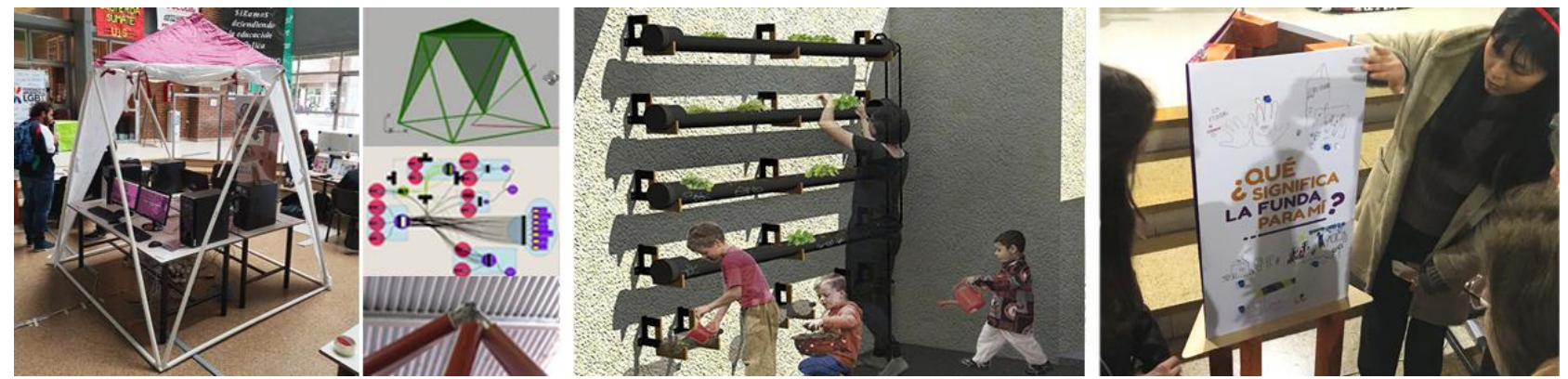

Figura 3: De izquierda a derecha: Pabellon fun.cional (G4); Jardín vertical (G5); Panel testimonial interactivo (G6). Fuente: autores.

\section{RESULTADOS}

Todas las producciones conformaron una propuesta integral que incluyó el diseño y producción del sitio web institucional; una aplicación para la organización interna; juegos de mesa para aprender el valor de una alimentación saludable; el mapeo colectivo del barrio; banners con realidad aumentada; estructuras modulares creadas a partir de diseño paramétrico; paneles interactivos; videos testimoniales e institucionales; y mobiliario lúdico. A continuación, se describirá brevemente cada trabajo.

Grupo 1. Sitio web institucional. Integrantes: Leal, Ranzuglia, Schneider.

De las problemáticas planteadas por las voluntarias de la Asociación, el grupo optó por trabajar en la comunicación externa. La propuesta consiste en una página web adaptativa o responsiva en la que se comunica, informa e invita a la sociedad a conocer de qué trata y que tipo de actividades realiza la institución. También constituye un medio para contactarse con potenciales voluntarios y donantes. La página se resuelve en WordPress utilizando código CSS para editar la estética, las tipografías, colores, etc., de la plantilla elegida, y vincularla con la identidad de la Asociación (Fig. 2). La plataforma elegida resulta fácil de actualizar por los voluntarios.

Grupo 2. ROMPEXA, rompecabezas didáctico. Integrantes: Castella, Duré, Giacopuzzi.

El grupo se propuso atender a la falta de material educativo para el desarrollo de talleres. Proponen un juego didáctico con información nutricional sobre distintos alimentos, que posibilita diferentes articulaciones geométricas (Fig. 2). ROMPEXA es un rompecabezas conformado por un hexaedro de color violeta y seis pentágonos de colores azul, verde, amarillo, naranja, celeste y rojo, con un total de 27 piezas que se pueden combinar para lograr diversas figuras de manera intuitiva, para fomentar el pensamiento creativo de niños y niñas. Para el diseño y modelado se utilizó Autocad y SketchUp, para los dibujos grabados CorelDraw, Adobe Illustrator y Photoshop, y para el corte laser del MDF se utilizó el software Autolaser.

Grupo 3. Banners con Realidad Aumentada. Integrantes: Ballestero, Castro, Signorelli, Torres.

La propuesta del grupo buscó potenciar la identidad visual de la Asociación Civil, estableciendo pautas gráficas de reproducción para distintas piezas institucionales con el objetivo de captar nuevos voluntarios, padrinos y colaboradores. Con este fin, planificaron una campaña de difusión mediante banners para su exhibición en los eventos a los que asista la institución, con la incorporación de marcadores de Realidad Aumentada (RA) que, al ser escaneados, redirigen al usuario a audiovisuales creados especialmente para la campaña (Fig. 2). Se procuró reforzar la identidad y darle visibilidad al rol social de la institución a partir del uso de metáforas, imágenes, códigos visuales y una gama cromática común en todas las piezas.

Grupo 4. Pabellón Fun.cional. Integrantes: Carrara, De Ángeli, Finós, Sakaziri Laburu, Vagni.

Este grupo se propuso aportar una solución al problema del espacio físico insuficiente, tanto para la realización de talleres como para la presentación en eventos. A partir del modelado paramétrico con Grasshopper, diseñaron una estructura fácil de montar y trasladar, liviana y modular, asignando como parámetros variables todas las dimensiones de la misma (altura, ancho, grosor de los caños, etc.). Esto les permitió reajustar el diseño fácilmente en base a los espesores y largos de los caños disponibles en el mercado, para optimizar el corte de los mismos y abaratar costos. En su construcción se utilizaron caños de PVC de $32 \mathrm{~mm}$ de diámetro exterior y $25 \mathrm{~mm}$ de diámetro interior, que se encastran a través de uniones metálicas soldadas. La cubierta se resuelve en Tela Silver (impermeable), con los colores identitarios de la Asociación, violeta el techo y blanco sus laterales (Fig.3).

Grupo 5. Jardín vertical "Verde esperanza". Integrantes: Amaya, Giménez, Gross.

A partir del deterioro ambiental del barrio, detectado por el grupo como problemática, se propone un jardín vertical para la sede de la Asociación Civil, que además de mejorar el aspecto edilicio, permita abordar la enseñanza y aprendizaje de valores sustentables, del trabajo en equipo, de la responsabilidad y constancia en el desarrollo de una tarea, dado que son los chicos del barrio quienes cultivarán las plantas. Con modelado en SketchUp, se diseña un sistema que combina "contenedores" hechos con caños de PVC y botellas plásticas recicladas; soportes de madera realizados con cortadora láser; y anclajes metálicos para colgar el jardín de la pared. El riego es automatizado a partir de una electroválvula y un temporizador (Fig. 3).

Grupo 6. "Panel testimonial interactivo". Integrantes: Carboni, Chen, Visgarra.

Al igual que el grupo 1, este grupo propone dar respuesta a la problemática de la comunicación de la asociación hacia el exterior. Para esto, desarrollan un dispositivo para visibilizar el trabajo de la institución y sensibilizar a la 


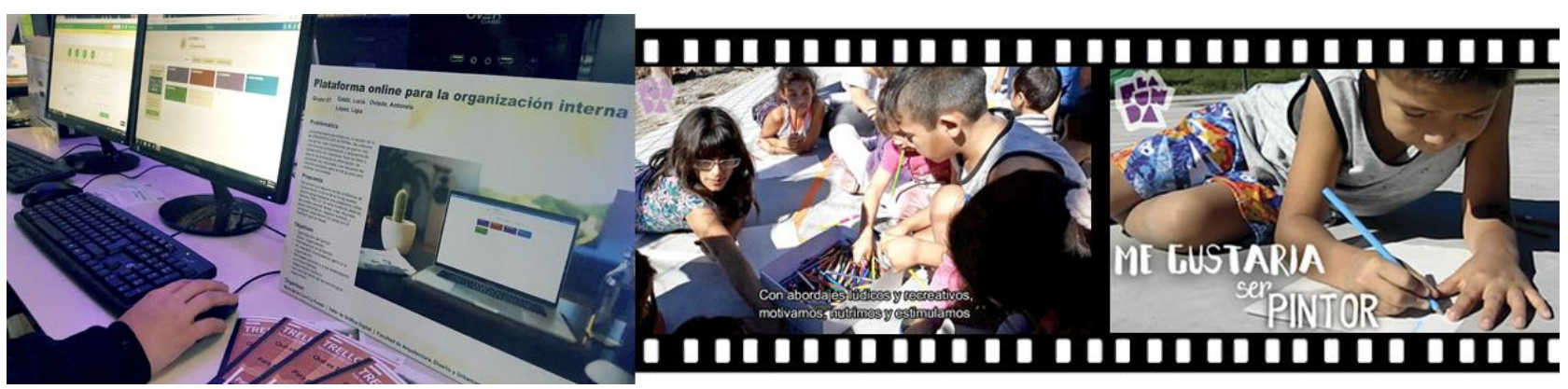

Figura 4: De izquierda a derecha: Plataforma online de gestión interna (G7); Video institucional (G9); Audiovisual "Cuando sea grande" (G8). Fuente: autores.

población. La propuesta se resuelve con un prisma de madera de base triangular, apoyado sobre 3 patas encastradas (Fig. 3), que incluye un sistema interactivo de luces y audio a partir del módulo reproductor de audio mp3 Arduino y bocinas de parlantes de 8 ohmios, que reproducen las voces de los niños/as respondiendo a la pregunta ¿qué es La Funda para vos? Presionando los botones analógicos integrados a un circuito impreso con dibujos de los niños/as, se encienden luces led y se disparan los audios.

Grupo 7. Plataforma online de gestión interna. Integrantes: Gabbi, López, Oviedo.

Como principal problemática a resolver, el grupo detectó la comunicación interna entre los miembros de la institución, que se relaciona con la manera de gestionar y compartir la información. La dispersión de la información en distintos soportes y formatos, tanto digitales como analógicos, dificultaba la comunicación y organización de las actividades. El grupo investigó las tecnologías disponibles para adaptarlas a las necesidades de la institución y lograr una mayor eficiencia en la organización, además de posibilitar el acceso remoto a la información, que permite un seguimiento continuo. Analizadas las variables: adaptabilidad a diferentes artefactos, sistema de visualización de aportes, gestión de usuarios y permisos, y posibilidad de personalización, se determinó que la plataforma más adecuada para gestionar la comunicación interna de la institución era Trello, una aplicación gratuita que se personalizó de acuerdo a las necesidades (Fig. 4).

Grupo 8. Audiovisual "Cuando sea grande". Integrantes: De Carolis, Delgado, García.

El objetivo de la propuesta fue difundir el rol social de la Asociación Civil mediante la realización de un video que muestra las aspiraciones y pensamientos de los niños y niñas que participan de sus actividades y talleres (Fig. 4). Con la pregunta ¿qué querés ser cuando seas grande? el objetivo fue captar la espontaneidad de los niños haciendo actividades dentro de su propio entorno, revelando la riqueza y ambigüedad de su medio, como forma de promover valores y el interés de la sociedad. En palabras del grupo, su objetivo fue explorar "la capacidad de las TIC para cruzar las fronteras sociales que nos segregan en clases". Estos videos fueron editados con diferentes formatos adaptados a las distintas redes sociales, para que trascienda a la sociedad la tarea que realiza la institución.

Grupo 9. Video Institucional. Integrantes: Farias, Oreggioni, Ricotti.
En base a las problemáticas diagnosticadas, este grupo decidió plantear una estrategia de comunicación para divulgar y dar a conocer la institución, mediante la realización de un video institucional que muestra el trabajo que realizan los integrantes, sus objetivos y sus logros. A través de la consigna "¿Sumas tu abrazo?" se buscó transmitir emociones, valores y sentimientos (Fig. 4), a fin de captar el interés para incorporar voluntarios y recursos mediante el uso de los medios de comunicación, principalmente redes sociales, intentando llegar a la mayor cantidad de público. La estética utilizada y la continuidad de la identidad gráfica, en relación a todas las piezas diseñadas para los distintos medios narrativos, manifiestan una consistencia del relato y sus modos de representación.

Grupo 11. Mapeo colectivo del barrio. Integrantes: Díaz, Mendoza Valencia, Sponton, Lizárraga, Morandin.

Este grupo realizó el mapeo del barrio donde funciona la Asociación Civil, sobre 4 categorías: salud, infraestructura, identidad y seguridad, para dar a conocer a toda la ciudadanía las condiciones reales del sector. Estos datos, previamente sistematizados, se volcaron y relacionaron utilizando p5.js, una biblioteca de JavaScript muy similar al lenguaje Processing, lo que resulta en una interfaz dinámica que muestra una red de relaciones entre los diferentes nodos relevados, brindando información sobre cada uno de ellos (textos, imágenes y videos). El mapeo posibilita visibilizar el territorio (Fig. 5), identificar problemáticas y reflexionar sobre conexiones con otras temáticas, y más allá de los entornos virtuales, asume lo territorial como instancia posible para narrar.

Grupo 12. Sistema de registro y Base de datos. Integrantes: Caravita, Girardo, González.

El grupo desarrolla un sistema online que facilita las tareas de registro, seguimiento y organización de las personas y familias vinculadas a la Asociación Civil. El proceso se desplegó en 2 etapas: 1) organización modular de la base de datos a partir del cuestionario que la institución utiliza para relevar el perfil personal de cada niño/a, su historial médico y nutricional, alimentación y área psicosocial; 2) búsqueda y selección del sistema adecuado. Para ello se compararon diversas alternativas de plugins (Ninja Forms, Contact Form 7, Staff List) y plataformas (Google Forms, Survey Monkey, Survio, Type Form). Finalmente se optó por Survey Monkey (Fig. 5), por su flexibilidad en el manejo y configuración de las preguntas y los datos, además de que permite exportar estadísticas generales y particulares. 


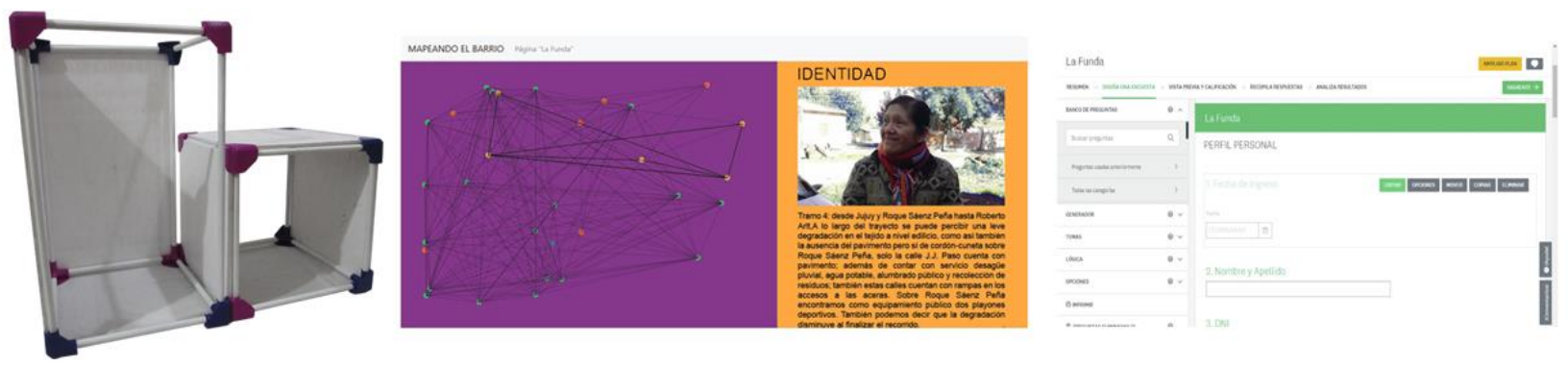

Figura 5: De izquierda a derecha: Mobiliario lúdico (G13); Mapeo colectivo del barrio (G11); Sistema de registro y base de datos (G12)

Grupo 13. Mobiliario lúdico "CUDICO". Integrantes: De Césaris, Oldani, Suárez, Uriarte.

La propuesta busca dar respuesta a la falta de mobiliario adecuado para niños, pero atendiendo a su vez a la carencia de espacio de la sede de la institución. Se diseña una línea de sillas y mesas desarmables, polivalentes, versátiles, económicas, livianas y fáciles de manipular por los niños/as, que admiten diferentes disposiciones y combinaciones. Luego de un largo proceso que incluyó el análisis de variables como la resistencia, el peso y la forma, los muebles fueron diseñados a partir de elementos estándar (caños metálicos de $1 / 2$ " y placas fenólicas de $6 \mathrm{~mm}$ ), unidos con conectores modelados digitalmente e impresos en 3D con PLA, un polímero biodegradable con mayor dureza, rigidez, y más fácil de imprimir que el ABS porque requiere menor temperatura de extrusión (Fig. 5).

Como se anticipó, la entrega final de los trabajos se realizó en una jornada de presentación y fundamentación de las propuestas, de la que participaron estudiantes, docentes y representantes de la Asociación Civil. Utilizamos dos sistemas de evaluación orientados al aprendizaje: la evaluación continua durante el proceso y la co-evaluación al finalizar, que promedió la autoevaluación de cada grupo con las notas asignadas por los docentes, para producir una única calificación que -en el $90 \%$ de los casos- fue superior a la autoevaluación.

Las producciones del Taller de Gráfica Digital 2018 se expusieron en una muestra realizada en el hall de ingreso a la facultad (Fig.1), donde además de la exhibición de los proyectos se pudo dar visibilidad al trabajo realizado por la Asociación Civil La Funda.

\section{DISCUSION}

A fin de realizar un estudio comparativo de los diferentes tipos de abordaje, los procesos desplegados y los resultados alcanzados a través de la experiencia, se recurre a categorías de análisis semiótico resignificadas por Güerri (2014) a partir del modelo tríadico de Pierce: FORMA (sintaxis y atributos de diseño), EXISTENCIA (factores materiales, estructurales y tecnológicos), y VALOR (contenidos, objetivos, significados pragmáticos y simbólicos). Dado que el sentido del proyecto general era colaborar con la Asociación Civil La Funda, el Valor fue una categoría determinante en la validación de los trabajos.

En las propuestas de los grupos 1, 2, 3 y 13, se destacan los valores de la Forma por sobre los atributos tecnológicos o semánticos, aunque el grupo 1 consideró todos los aspectos con igual intensidad. Los densos procesos de diseño, el gran nivel de detalle que lograron los trabajos, el apropiado uso de colores y metáforas visuales, así como la búsqueda de formas más eficientes en relación a los materiales y las actividades, caracterizan a los artefactos, interfaces y representaciones de estos grupos.

Por su parte, en las propuestas de los grupos 4, 5, 11 y 12 , en el abordaje del problema se han priorizado los aspectos tecnológicos y de materialización, vinculados a la Existencia. Estos grupos se destacan por las habilidades demostradas para resolver cuestiones vinculadas al manejo de los softwares y los lenguajes de programación, a la utilización de materiales y técnicas de fabricación, al diseño de estructuras y conectores, y a los niveles de terminación de los productos.

En tanto los grupos 6, 7, 8 y 9 desarrollaron ideas y proyectos que resaltan los aspectos vinculados al Valor, condición que se manifiesta en la trascendencia de los contenidos y objetivos, la expresividad y originalidad de los argumentos, la riqueza performativa o interactiva de las propuestas, la utilidad de las aplicaciones o la consistencia emotiva y simbólica de los mensajes, que hacen del significado la mayor fortaleza de estos trabajos que constituyen valiosos aportes para la Asociación Civil.

\section{BALANCE ENTRE EMOCIÓN Y COGNICIÓN}

Días previos a la entrega final, se realizaron encuestas dirigidas a estudiantes y a docentes, orientadas a relevar el impacto generado por la experiencia educativa. Éstas se realizaron con formularios de Google e incluyeron preguntas cerradas y abiertas.

En base a las opiniones relevadas en las encuestas, podemos afirmar que la experiencia resultó positiva, no sólo por el nivel alcanzado por las producciones realizadas en el proyecto colectivo, sino también por el grado de satisfacción de los destinatarios. La propuesta transmedia, sostenida por las plataformas provistas para este fin, otorgó dinamismo y diversidad a los procesos de enseñanza y aprendizaje, particularmente en lo que respecta a la participación de los estudiantes, además de establecer un feedback fluido entre docentes y estudiantes.

Los procesos de aprendizaje colectivos demandan el establecimiento y sostenimiento de vínculos personales activos entre los actores educativos, y un gran compromiso por parte de todos. Se trata de crear una conciencia de grupo, valorando y recompensando la cooperación que, en tanto método para la consecución de un objetivo común, incentiva la participación, la responsabilidad compartida y la autogestión. 
Las estrategias didácticas reticulares, donde todos los actores se constituyen en coautores solidarios, favorecen la conformación de comunidades educativas en red que se enmarcan en una cultura de aprendizaje a lo largo de la vida y comprenden todas las actividades, medios y ámbitos relativos a la educación.

El balance entre innovación morfológica, destreza técnica, responsabilidad, compromiso y desarrollo de actitudes colaborativas, se basa en el entrelazamiento de factores cognitivos y afectivos que guían tanto el comportamiento racional y la toma de decisiones, como la creatividad. Un adecuado equilibrio entre emoción y cognición, es una condición necesaria para lograr aprendizajes significativos.

\section{REFLEXIONES FINALES}

Debido a que las tecnologías digitales son medios y no fines, fue posible abordar una multiplicidad de problemáticas sociales y propuestas de diseño que demandaron una diversidad de recursos, así como una gran capacidad de adaptación a situaciones emergentes e imprevisibles, y si bien la articulación con el medio resultó un factor motivante de aprendizajes significativos, las condiciones del contexto a su vez acotaron ciertos aspectos de las propuestas, como la posibilidad de desarrollar proyectos tecnológicos complejos o que requiriesen instrumentos de última generación para activarlos o reproducirlos.

Más allá de esto, la experiencia confirmó que es posible una integración efectiva entre educación, investigación y vinculación con el medio, para idear y poner en acto respuestas creativas a problemas sociales y ambientales del territorio, con un sentido de comunidad que impulse la construcción de una sociedad más abierta e inclusiva.

El espacio aumentado debe adecuarse a usos emergentes y significados variables en relación a diferentes grupos sociales y no ser determinado sólo por sus creadores. Una participación social activa en el diseño y desarrollo del espacio aumentado es clave, ya que la apropiación que realizan los grupos sociales es resultado de procesos de resignificación de carácter dialógico y plural.

Según el enfoque dialógico de Freire (1989), un sistema distribuido de construcción de conocimientos posibilita interactuar con la cultura existente, así como recrearla de modo crítico y emancipador, como un derecho y una necesidad de los ciudadanos de la sociedad informacional.

"Eso es lo que significa la palabra "emancipación": el borramiento de la frontera entre aquellos que actúan y aquellos que miran, entre aquellos que saben y aquellos que no, son historias de fronteras a ser cruzadas y de distribuciones de roles a borrar..." (Rancière, 2010: 2526).

\section{AGRADECIMENTOS}

Agradecemos la constante colaboración de: Mg. Arq. Matías Dalla Costa; LCV Agustín Longoni; tesistas: Verónica Rainaudo, Micaela Block y Silvana Fontana; Federico Carrara y los padres de algunxs estudiantes.
También agradecemos a la Universidad Nacional del Litoral por financiar el proyecto de investigación "Mediaciones del Espacio Aumentado", y destacamos la valiosa participación de las representantes de la Asociación Civil "La Funda”: Luz Carrara y Luisina Berca.

\section{REFERENCIAS}

Bermudez, J. (1995). Designing Architectural Experiences: Using Computers to Construct Temporal 3D Narratives. ACADIA Conference Proceedings. Seattle: University of Washington, pp. 139-149.

Collaborative Learning Enhances Critical Thinking http://scholar.lib.vt.edu/ejournals/JTE/v7n1/gokhale.jtev7n1.html?ref=Sawos. Org

Combes, L. (1997). Dibujo ayudado por computadora vs. Diseño ayudado por computadora. 1er. Seminario de Gráfica Digital, Vol. 1. Ed. CEADIG: Buenos Aires, pp. 9-13

Freire, P., Macedo, S. (1989). Alfabetización. Lectura de la palabra y lectura de la realidad. Madrid: Paidos/MEC.

Güerri, C. (2014). Nonágono Semiótico un modelo operativo para la investigación cualitativa. Buenos Aires: Eudeba.

Habermas, J. (1989). Teoría de la Acción Comunicativa. Madrid: Ediciones Cátedra.

Huxley, A. (1932) [2014]. Un mundo feliz. Madrid: DeBolsillo.

Kozak, C. (2011). Tecno-poesía experimental y políticas del acontecimiento. En Poéticas tecnológicas, transdisciplina y sociedad. Buenos Aires: Exploratorio Ludión.

Kranzberg, M., Davenport, W. (1972) [1979]. Technology and culture. An anthology. New York: Schocken Books.

Levy, P. (1998). Becoming Virtual: Reality in the Digital Age. New York: Da Capo Press.

Machado, A. (2000). Repensando a Flusser y las imágenes Técnicas. En El paisaje mediático. Buenos Aires: Libros del Rojas.

Marcuse, H. (1993). El hombre unidimensional. Ensayo sobre la ideología de la sociedad industrial avanzada. Buenos Aires: Planeta Agostini

Mitchell, W. (1990). The logic of architecture. Cambridge: Massachusetts Institute of Technology Press.

Montagú, A. (1996). Seminario de Investigación. Centro de Informática y Diseño. Ciudad X: Universidad X

Novak, M. (1991). Liquid Architectures in Cyberspace. En: Benedikt, M., ed., Cyberspace: First Steps, Cambridge: The MIT Press, pp. 226-249.

Picard, R., Papert, S., Bender, W., Blumberg, B. (2004). Affective Learning - A Manifesto. Journal BT Technology Journal Volume 22 Issue 4, pp. 253-269

Rancière, J. (2010). El Espectador Emancipado. Buenos Aires: Manantial.

Scolari, C. (2008). Hipermediaciones, Elementos para una Teoría de la Comunicación. Barcelona: Gedisa.

Senegala, M. (1999). An Epistemological and Systems Approach to Digital Technology Integration in Architectural Curriculum. In Media and Design Process. Salt Lake City: ACADIA '99. http://papers.cumincad.org/data/works/att/c05b.content.pdf

Thomas, H., Buch, A. (2008). Actos, Actores y Artefactos. Buenos Aires: Universidad Nacional de Quilmes Editorial.

Tosello, M. (1997). El universo digital dentro del taller de Arquitectura. 1er. Seminario de Gráfica Digital, Vol. 2. Ed. CEADIG: Buenos Aires, pp. 81-86

Tosello, M. (2002). El ambiente digital. Vacío y multiplicidad. 6to. Congreso de Gráfica Digital. Caracas, Venezuela. pp.43-46. http://papers.cumincad.org/data/works/att/394e.content.pdf 